Pacific Journal of Mathematics

SECONDARY COHOMOLOGY OPERATIONS WHICH EXTEND 


\title{
SECONDARY COHOMOLOGY OPERATIONS \\ WHICH EXTEND THE TRIPLE PRODUCT
}

\author{
DONALD W. KAHN
}

The triple product of Massey [5] is a secondary cohomology operation in three variables which is defined when the three classes satisfy two relations. The value of the operation is a certain natural coset of cohomology. In this note, I discuss certain secondary operations which generalize the triple product, when the values are taken in cohomology with real coefficients. Roughly speaking, for each pair of integers $(M, N)$, there is such an operation; when one takes the pair $(1,1)$, the operation is the triple product.

There are four sections. The first section, which is primarily motivation, discusses operations in real cohomology. In this section, explicit cochain formulas are given. In the remaining sections, we are concerned with operations whose domain is integral cohomology, and whose range is real cohomology. The second section studies the universal examples. In the third section, I define the operations and determine their ranges. Finally, in the fourth section, I prove a classification theorem for these operations, which asserts that in certain cases these operations constitute all such operations.

I would like to thank W. S. Massey and N. Stein for their help and encouragement during the preparation of this paper.

1. Consider now operations in real cohomology. We restrict attention to spaces which are 1-connected, countable simplicial complexes. In [4] the author showed that for such spaces, there is an anti-commutative cochain algebra over the real numbers, which gives the correct cohomology. If $X$ is a space in our category, write this anti-commutative cochain algebra as $\mathscr{G}(X)$.

The Massey triple product may be defined as follows: consider classes $u, v, w \in H^{*}(X, R)^{1}$ such that $u \cdot v=v \cdot w=0$. Choose

$$
u^{\prime}, v^{\prime}, w^{\prime} \in \mathscr{G}(X)
$$

which represent these classes, and $a, b \in \mathscr{G}(X)$ such that

$$
u^{\prime} \cdot v^{\prime}=\delta a, v^{\prime} w^{\prime}=\delta b
$$

Set

$$
Z^{\prime}=a \cdot w^{\prime}-(-1)^{\operatorname{Dim}(u)} u^{\prime} \cdot b
$$

Received July 9, 1962. Work was supported in part by contract NONR 266 (57).

1 Real cohomology is to be understood in this section. 
One easily sees that $\delta Z^{\prime}=0$. Depending on the different choices, $Z^{\prime}$ represents a set of classes in $H^{*}(X)$ (in fact a coset).

Now, using the anti-commutativity of $\mathscr{G}(X)$, we make the following definition.

Definition 1.1. (Double Product). Let $u \in H^{p}(X), v \in H^{q}(X)$. Suppose $q$ is odd, and $u \cdot v=0$. Choose $u^{\prime}, v^{\prime} \in \mathscr{G}(X)$, representing $u$ and $v$. Let $u^{\prime} \cdot v^{\prime}=\delta a$.

Define

$$
\begin{gathered}
Z^{\prime}=a \cdot v^{\prime} \\
\delta\left(a \cdot v^{\prime}\right)=u^{\prime} \cdot v^{\prime} \cdot v^{\prime}=u^{\prime} \cdot\left(v^{\prime}\right)^{2}=0, \text { as }\left(v^{\prime}\right)^{2}=0 .
\end{gathered}
$$

Set $Z=\left\{Z^{\prime}\right\}$, and $\langle u, v\rangle=\{Z\}$, where the latter is the set of all classes represented by such $Z$. $\langle u, v\rangle$ is the double product of $u$ and $v$.

Definition 1.2. (Generalized Double Product). With the same conventions as Definition 1.1, we add the assumption that $p$ is even. Define $Z^{\prime}=a^{n} \cdot v^{\prime}, n$ being positive integer.

$$
\delta Z^{\prime}=\left(n \cdot a^{n-1} \cdot u^{\prime} v^{\prime}\right) v^{\prime}=0 .
$$

Set $Z=\left\{Z^{\prime}\right\}$, and $\langle u, v\rangle_{n}=\{Z\},\langle u, v\rangle_{n}$ is the generalized double product.

Definition 1.3. (Generalized Triple Product). Consider classes $u, v, w \in H^{*}(X)$ in dimensions $p, q, r$ (resp.). Suppose $p$ and $r$ are odd. (If one is even, one may choose $k$ or $l$ below to be 1). Suppose $u \cdot v=v \cdot w=0$. Choose $u^{\prime}, v^{\prime}, w^{\prime} \in \mathscr{G}(X)$ representing $u, v, w$, and choose $a, b \in \mathscr{G}(X)$ so that

$$
u^{\prime} \cdot v^{\prime}=\delta a \quad v^{\prime} \cdot w^{\prime}=\delta b .
$$

Let $k$ and $l$ be positive integers. Set

$$
Z^{\prime}=l \cdot a^{k} w^{\prime} b^{l-1}-(-1)^{k(p+q-1)+(q-1)} k \cdot a^{k-1} u^{\prime} \cdot b^{l} .
$$

An elementary computation shows that $\delta Z^{\prime}=0$. Let $Z=\left\{Z^{\prime}\right\}$, and $\langle u, v, w\rangle_{k, l}=\{Z\}$, the generalized triple product.

REMARKs. One easily computes the dimensions for these operations. For example,

$$
\operatorname{dim}\langle u, v, w\rangle_{K, l}=k \cdot p+(k+l-1)(q-1)+l \cdot r .
$$

The double product $\langle u, v\rangle$ is quite similar to the triple product $\langle u, v, v\rangle$. However, the denominator modulo which it is defined is 
actually smaller than the corresponding denominator for the triple product.

Finally,

Definition 1.4. With the notations of Definition 1.3, suppose dim. $v$ is odd.

Let

$$
Z^{\prime}=v^{\prime} \cdot a^{m} \cdot b^{n}, \quad\left\{Z^{\prime}\right\}=Z
$$

Let

$$
{ }_{m, n}\langle u, v, w\rangle=\{Z\}
$$

REMARKS. It is clear that these operations are generalizations of the triple product (in real cohomology). However, they do not lend themselves to computation, because of the difficulty of computing in $\mathscr{G}(X)$. Therefore, in order to obtain a fuller theory, the remainder of this paper will study similar operations which have integral cohomology as domain and real cohomology as range. The technique of universal examples can then be applied.

We shall assume that all spaces have the homotopy type of a 1-connected countable simplicial complex.

2. We now give the basic definitions and theorems for universal examples. A primary operation in one variable is a natural function

$$
\varphi: H^{p}(X, G) \longrightarrow H^{q}(X, H)
$$

where $p$ and $q$ are positive integers, and $G$ and $H$ are Abelian groups. Such an operation is said to be of type $\left\{p, G_{j q}, H\right\}$. Serre [9] and others observed that the cohomology of Eilenberg-MacLane spaces described all such operations. Precisely, there is a natural one to one correspondence between operations of type $\{p, G ; q, H\}$ and classes of $H^{q}(G, p ; H)$. If $\varphi$ is of type $\{p, G ; q, H\}$ then this correspondence assigns to $\varphi$ the class $\varphi(i)$, where $i$ is the fundamental class of $H^{p}(G, p ; G)$. Hence, a knowledge of operations is equivalent, in principle, to a knowledge of the operations in a "universal example", here a space $K(G, p)$.

The theory of universal examples may be extended to cover operations in several variables, and higher order operations, which are defined when certain operations vanish, and which take on a values certain sets of elements in the cohomology of a space. In the definition of such operations, one imposes only one condition, naturality. For a general discussion of such operations, and the corresponding universal examples, we refer to [1], [3], and [7]. The information 
which we shall need is contained in the following two theorems.

Theorem 2.1. Let $X$ be a space in our category. The universal example for a primary operation $\varphi$, in the variables $u_{i} \in H^{p_{i}}\left(X, G_{i}\right)$ is a space

$$
K\left(G_{1}, p_{1}\right) \times \cdots \times K\left(G_{n}, p_{n}\right) .
$$

That is, the operations $\varphi\left(u_{1}, \cdots, u_{n}\right) \in H^{q}(X, H)$ are in one-to-one correspondence with the classes of

$$
H^{q}\left(K\left(G_{1}, p_{1}\right) \times \cdots \times K\left(G_{n}, p_{n}\right) ; H\right) .
$$

Theorem 2.2. Consider $\mathrm{X}$ and $u_{i}$ as above. Suppose that the classes satisfy the homogeneous distinct relations

$$
P_{1}\left(u_{1}, \cdots, u_{n}\right)=0, \cdots, P_{m}\left(u_{1}, \cdots, u_{n}\right)=0
$$

where we have associated to each relation, a pairing of the groups $G_{1}, \cdots, G_{n}$ to a group $A_{i}$.

Then, the universal example for operations in these variables, defined when these relations are satisfied (i.e., on the kernel of the operations $\left.P\left(u_{1}, \cdots, u_{n}\right), \cdots, P_{m}\left(u_{1}, \cdots, u_{n}\right)\right)$, is a fibre space $(E, F, B ; P)$, with

$$
\begin{gathered}
B=K\left(G_{1} ; p_{1}\right) \times \cdots \times K\left(G_{n} ; p_{n}\right) \\
F=K\left(A_{1} ; \operatorname{deg} . P_{1}-1\right) \times \cdots \times K\left(A_{m} ; \operatorname{deg} . P_{m}-1\right) .
\end{gathered}
$$

The structure of the fibre space is determined by a map

$$
f: B \longrightarrow K\left(A_{1}, \operatorname{deg} . P_{1}\right) \times \cdots \times K\left(A_{m} ; \operatorname{deg} . P_{m}\right)
$$

which is given by the product of the relations. That is, such operations $\varphi\left(u_{1}, \cdots, u_{n}\right) \in H^{q}(X, H)$ are in one to one correspondence with the classes of $H^{q}(E, H)$.

The correspondence is as follows: Write $u_{1}, \cdots, u_{n}$ as maps

$$
X \stackrel{u_{1}}{\longrightarrow} K\left(G_{1}, p_{1}\right), \cdots, X \stackrel{u_{n}}{\longrightarrow} K\left(G_{n}, p_{n}\right) .
$$

If the classes $u_{1}, \cdots, u_{n}$ satisfy the relations, then the composition

$$
\begin{aligned}
X \stackrel{u_{1} \times \cdots \times u_{n}}{\longrightarrow} K\left(G_{1}, p_{1}\right) \times \cdots \times K\left(G_{n}, p_{n}\right) \stackrel{f}{\longrightarrow} & K\left(A_{i}, \operatorname{deg} . P_{1}\right) \\
& \times \cdots \times K\left(A_{m}, \operatorname{deg} . P_{m}\right)
\end{aligned}
$$

is homotopic to zero. Hence, the map $u_{1} \times \cdots \times u_{n}$ may be lifted to a map $U: X \longrightarrow E$, so that the following diagram is commutative. 


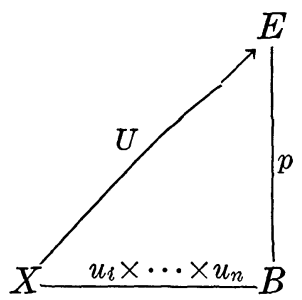

If $\varphi \in H^{q}(E, H)$, we define $\varphi\left(u_{1}, \cdots, u_{n}\right)$ to be the collection $\left\{U^{*}(\varphi)\right\}$ in $H^{q}(X, H)$ where $U$ ranges over all lifting maps which make the above diagram commutative.

Proof. See the references mentioned above. The proofs of these theorems follow immediately from the techniques of those paper.

Conventions. For the remainder of this paper, assume that all operations go from integral cohomology. That is, the $G_{i}$ and $A_{i}$ are always $Z$, while $H$ is $R$. The pairings will always be the natural multiplication in $Z$. The cohomology of a space will be real singular cohomology.

I will now indicate how the above theorems apply to the cupproduct, and to the triple product [5].

(a) If we were to choose as coefficients any ring with identity, the cup-product is an operation in two variables, given by the composition

$$
H^{p}(X) \otimes H^{q}(X) \longrightarrow H^{p+q}(X \times X) \stackrel{\Delta^{*}}{\longrightarrow} H^{p+q}(X)
$$

where $\Delta$ is the diagonal. We shall consider the particular cup-product.

$$
H^{p}(X ; Z) \otimes H^{q}(X ; Z) \longrightarrow H^{p+q}(X ; R)
$$

This operation is described in terms of the universal example as follows. The space involved is $K(Z, p) \times K(Z, q)$. By the Kunneth theorem:

$$
H^{p+q}(K(Z, p) \times K(Z, q) ; R) \underset{0 \leqq i \leqq p+q}{\approx} H_{i}(Z, p ; R) \otimes H^{p+q-i}(Z, q ; R) .
$$

Let $i_{p}$ and $i_{q}$ be the generators of $H^{p}(Z, p ; R)$ and $H^{q}(Z, q ; R)$, i.e., the images, under the coefficient homomorphism $Z \rightarrow R$, of the fundamental classes. Then, our cup-product $(*)$ corresponds to the class $i_{p} \otimes i_{q}$.

More generally, we have the following.

Proposition 2.1. Consider classes

$$
u_{1} \in H^{p_{1}}(X, Z), \cdots, u_{n} \in H^{p_{n}}(X, Z),
$$

where $X$ is some space in our category. Then, all primary operations. 
in these classes, which take on values in real cohomology, are given by (formal) polynomials in these classes, with respect to cup-product, that is linear combinations of monomials with respect to cup-product multiplication.

Proof. One simply computes the real cohomology of the universal example, which is

$$
K\left(Z, p_{1}\right) \times \cdots \times K\left(Z, p_{n}\right) .
$$

(b) We define the triple product. Consider three cohomology classes $u \in H^{p}(X ; Z), v \in H^{q}(X, Z), w \in H^{r}(X, Z)$. Suppose that these classes satisfy the relations

$$
u \cdot v=0 \quad v \cdot w=0 \quad \text { (pairing } Z \otimes Z \rightarrow R \text { ). }
$$

Choose representatives $u^{\prime}, v^{\prime}, w^{\prime}$ and cochains $a$ and $b$ so that

$$
u^{\prime} \cdot v^{\prime}=\delta a \quad v^{\prime} \cdot w^{\prime}=\delta b .
$$

We set

$$
J^{n}(u, w)=u \cdot H^{n-p}(X)+H^{n-r}(X) \cdot w \quad \text { (real cohomology) }
$$

where

$$
n=p+q+r-1 \text {. }
$$

Define

$$
Z^{\prime}=a \cdot w^{\prime}-(-1)^{p} u^{\prime} \cdot b \quad \text { (pairing } Z \otimes Z \rightarrow R \text { ). }
$$

One easily checks that $\delta Z^{\prime}=0$, and that $\left\{Z^{\prime}\right\}$ represents a coset of $H^{n}(X) / J^{n}(u, w)$. This coset is defined to be the triple product $\langle u, v, w\rangle$. When no confusion is possible, we shall not distinguish between $\langle u, v, w\rangle$ and $\left\{Z^{\prime}\right\}$.

Using Theorem 2.2, we construct a universal example in the case which we are considering. It is a fibre space $(E, F, B ; p)$, with

$$
\begin{aligned}
& B=K(Z, p) \times K(Z, q) \times K(Z, r) \\
& F=K(Z, p+q-1) \times K(Z, q+r-1) .
\end{aligned}
$$

The structure of the fibre space is given by a map

$$
B \longrightarrow K(Z, p+q) \times K(Z, q+r)
$$

representing the two cup-products.

Proposition 2.2. Consider the universal example for the triple product $(E, F, B ; p)$ Suppose $p<r$. Let $u \in H^{p}(Z, p)$ be a generator 
in the base, and $y \in H^{q+r-1}(Z, q+r-1)$ be a generator in the fibre ${ }^{2}$. Then $u \otimes y \in E_{2}$, in the Serre spectral sequence, remains until $E_{p}$, and represents the triple product $\langle u, v, w\rangle$ up to a sign.

If $p=r$, let $w \in H^{r}(Z, r)$ and $x \in H^{p+q-1}(Z, p+q-1)$ be generators for the base and fibre respectively. Then

$$
u \otimes y-(-1)^{r \cdot(p+q)} w \otimes x
$$

remains and represents the triple product up to a sign.

Proof. Suppose $p<r$. Consider the universal fibre space

$$
\begin{aligned}
& K(Z, p+q-1) \times K(Z, q+r-1) \longrightarrow \text { paths } \\
& \qquad K(Z, p+q) \stackrel{\downarrow}{\times} K(Z, q+r) .
\end{aligned}
$$

We use $x$ and $y$ to denote the generators in the fibre, also. It is well-known that $\tau(x)$ and $\tau(y)$ generate the cohomology of the base, and it follows from the naturality of the transgression that in the universal example $(E, F, B ; p), x$ and $y$ are transgressive and

$$
\tau(x)=u \cdot v \quad \tau(y)=v \cdot w
$$

where $u, v, w$ generate $H^{p}(Z, p), H^{q}(Z, q), H^{r}(Z, r)$ (resp.). Now consider the $E_{2}$-term of the spectral sequence of $(E, F, B ; p)$ (see [10]).

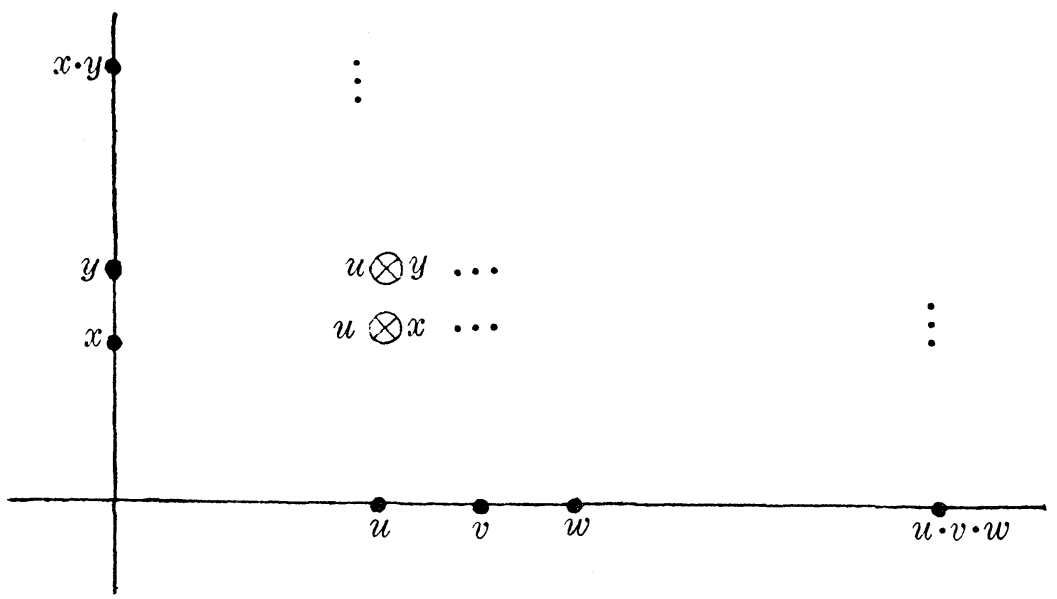

Let $n=p+q+r-1$. As $y$ is transgressive,

$$
d_{i}(u \otimes y)=0, i \leqq n-p, d_{n-p+1}(u \otimes y)=\{u \cdot v \cdot w\},
$$

where the brackets indicate the representation of an element in a

${ }^{2} \mathrm{We}$ take as generators the images of the generators in integral cohomology, under the map induced by the inclusion $Z \rightarrow R$. 
higher term of the spectral sequence. However, already

$$
d_{n-r+1}( \pm w \otimes x)=\{u \cdot v \cdot w\} \text {. }
$$

Hence $\{u \otimes y\}$ is a cocycle for all $d i$. One easily sees that $\{u \otimes y\}$ is not a coboundary for any $d_{i}$.

It is easy to see that $\{u \otimes y\}$ is the only element of dim. $n$ in $E_{\infty}$ so that it must represent some multiple of the triple product. We now make this determination more precise. Consider the classes $p^{*} u, p^{*} v$, and $p^{*} w$ in $H^{*}(E)$. Since $x$ and $y$ are transgressive, with $\tau(x)=u \cdot v, \tau(y)=v \cdot w$, we can choose cochains $C_{x}$ and $C_{y}$ in $C^{*}(E)$ such that

$$
\begin{array}{ll}
\delta C_{x}=p^{*} u^{\prime} \cdot p^{*} v^{\prime} & \left\{i^{*}\left(C_{x}\right)\right\}=x \\
\delta C_{y}=p^{*} v^{\prime} \cdot p^{*} w^{\prime} & \left\{i^{*}\left(C_{y}\right)\right\}=y
\end{array}
$$

with $u^{\prime} \cdot v^{\prime} \cdot w^{\prime}$ representing $u, v, w$. We have, by definition

$$
\left\langle p^{*} u, p^{*} v, p^{*} w\right\rangle=\left\{C_{x} \cdot p^{*} w^{\prime}-(-1)^{p} p^{*} u^{\prime} \cdot C_{y}\right\} .
$$

In the Serre spectral sequence, $C_{x}$ and $C_{y}$ represent elements in $E_{2}^{0 . *}$ corresponding to $x$ and $y$ in the fibre. It follows that the cochain $C_{x} \cdot p^{*} w^{\prime}-(-1)^{p} p^{*} u^{\prime} \cdot C_{y}$ represents in $E_{\infty}$ that element which comes from the term

$$
(1 \otimes x)(w \otimes 1)-(-1)^{p}(u \otimes y)
$$

in $E_{2} . \quad w \otimes x$ disappears by $E_{n-r+1}$. Up to a sign, the element $\{u \otimes y\}$ then determines the triple product.

The case where $p \geqq r$ is handled in a similar manner, and is left to the reader.

3. We now define the double product and generalized double product in terms of the universal example.

\section{DeFinition 3.1.}

(a) Double Product: Consider classes $u, v$; dim. $u=p>1$ dim. $v=q>1, q$ odd. Suppose that $u \cdot v=0$. The universal example for operations defined under these circumstances is a fibre space $(E, F, B ; p)$, with

$$
\begin{aligned}
& B=K(Z, p) \times K(Z, q) \\
& F=K(Z, p+q-1)
\end{aligned}
$$

${ }^{3}$ Concerning groups, real cohomology is to be understood, for the remainder of the paper. Operations will go from integral to real cohomology. 
with classifying map

$$
f: K(Z, p) \times K(Z, q) \longrightarrow K(Z, p+q)
$$

representing the cup-product, $u \cdot v$.

Consider the $E_{2}$-term in the spectral sequence for this fibre space. Let $u$ and $v$ generate $H^{p}(Z, p)$ and $H^{q}(Z, q)$ in the base, and $x$ generate $H^{p+q-1}(Z, p+q-1)$ in the fibre. As in Prop. 2. 2, $\tau(x)=u \cdot v$.

Clearly $d_{i} \equiv 0$ for $2 \leqq i<n=p+q$. We look at $d_{n}$.

$$
d_{n}(x)=u \cdot v . \quad d_{n}(u \otimes x)=u^{2} v . \quad d_{n}(v \otimes x)=0 .
$$

$\{v \otimes x\}$ is not a coboundary under $d_{n}$, as $d_{n}\left(E_{n-1}\right)$ is contained in the ideal generated by $\{u \cdot v \otimes 1\}$. Hence, $\{v \otimes x\}$ remains until $E_{\infty}$, and represents an element in $H^{*}(E)$. We define a class in $H^{*}(E)$, the double product, to be this element, and write, by abuse of language,

$$
\langle u, v\rangle=\{v \otimes x\} \text {. }
$$

(b) Generalized Double Product: We make the same assumptions as in (a), but also assume that $p$ is even. Then $p+q-1$ is even. We consider the universal example and note that if $n=p+q$, then $d_{i} \equiv 0$, for $2 \leqq i<n$. We compute $\operatorname{ker} d_{n}: d_{n}(x)=u \cdot v, d_{n}(u \otimes x)=$ $u^{2} \cdot v, d_{n}(v \otimes x)=0, d_{n}\left(x^{k}\right)=k \cdot u v \otimes x^{k-1}, d_{n}\left(u \otimes x^{k}\right)= \pm k \cdot u^{2} v \otimes x^{k-1}$, $d_{n}\left(v \otimes x^{k}\right)=0$. We may represent $E_{n+1}$ by the following picture, in which $\bullet$ indicates the presence of an element:

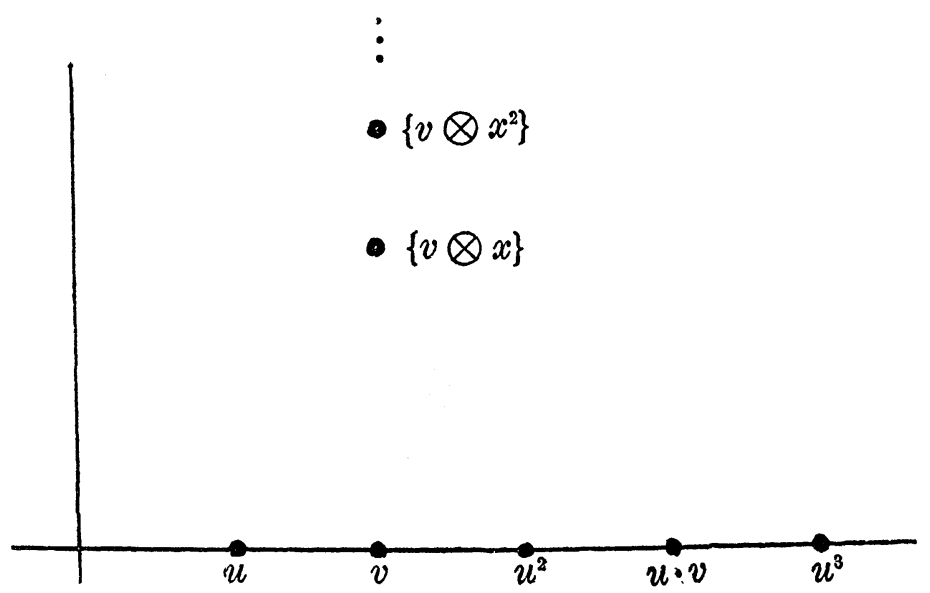

We now show that $\left\{v \otimes x^{k}\right\}, k \geqq 1$ remains until $E_{\infty}$. The difference in dimension between $u^{a}$ and $u^{a+1}$ is $p$. The difference in dimension between $v \otimes x^{k}$ and $v \otimes x^{k+1}$ is $p+q-1>p$. Hence, if $u^{a}$ (or $v \cdot u^{a}$ ) is killed off by $d_{i}$ of $\left(v \otimes x^{k}\right)$ for some $i$ and $k, u^{a+1}$ (or $v \cdot u^{a+1}$ ) cannot 
be killed. Hence, if we had $d_{i}\left(v \otimes x^{k}\right)=u^{a}$, then in $E_{\infty}$, we would have $u^{a}=0, u^{a+1} \neq 0$, which is absurd. Therefore, $\left\{v \otimes x^{k}\right\}$ represents a non-zero element in $E_{\infty}$, and we define, as before,

$$
\langle u, v\rangle_{k}=\left\{v \otimes x^{k}\right\} \text {. }
$$

It is clear that $\langle u, v\rangle_{1}=\langle u, v\rangle$.

REMARK. The operations $\langle u, v\rangle_{k}$ are clearly non-trivial. One simply computes them in $E$.

Next, I want to discuss an extension of the double product, which amounts to forming a double product in which each variable is actually a (formal) polynomial, with respect to the cup-product, of certain classes.

Consider classes $u_{1}, \cdots, u_{n}$, which satisfy a homogeneous relation $P\left[u_{1}, \cdots, u_{n}\right]=0$, where $P$ is a homogeneous-degree (formal) polynomial. The universal example for secondary operations, which are then defined, is a fibre space $(E, F, B ; p)$, with

$$
B=K\left(Z, \operatorname{dim} . u_{1}\right) \times \cdots \times K\left(Z, \operatorname{dim} . u_{n}\right)
$$

and

$$
F=K(Z, \operatorname{dim} . P-1) .
$$

Let $u_{1}, \cdots, u_{n}$ denote generators of $H^{*}(B)$, and $x$ a generator of $H^{*}(F)$. Then $\tau(x)=P\left[u_{1}, \cdots, u_{n}\right]$. Let $Q$ be a polynomial in the $u_{i}$, such that $P \cdot Q=0$, i.e., $P \cdot Q$ is forced to vanish by the anti-commutativity. Consider

$$
Q \otimes x^{k} \in E_{2} .
$$

Clearly, $d_{i}\left(Q \otimes x^{k}\right)=0,2 \leqq i<n=\operatorname{dim} . P$. But, also, $d_{n}\left(Q \otimes x^{k}\right)=$ $k \cdot Q \cdot P \otimes x^{k-1}=0$. Suppose that $Q \otimes x^{k}$ remains until $E_{\infty}$, and hence represents an element in $H^{*}(E)$. Then, we say that $Q \otimes x^{k}$ determines a generalized double product, and write

$$
\langle P \mid Q ; Q\rangle_{k}=\left\{Q \otimes x^{k}\right\} \text {. }
$$

REMARK. If there are $l$ independent generalized double products in some fixed dimension, then we can conclude that there are $l$ independent secondary operations which are defined on the kernal, $P=0$, and which take on values in the fixed dimension. In general, we cannot identify particular elements in $E_{\infty}$ with particular classes in $H^{*}(E)$.

The analogous statements also apply to the generalized triple products. Details are left to the reader.

We now determine the denominators, modulo which the double 
and generalized double products are defined.

Proposition 3.1. Consider a space $X$ in our category, and classes $u$ and $z$ in dimensions $p$ and $q$. Suppose $q$ is odd. Then

$$
\langle u, z\rangle \in H^{n}(X) / z \cdot H^{n-p}(X), n=p+2 q-1,
$$

and if $p$ is even,

$$
\begin{aligned}
\langle u, z\rangle_{k} \in H^{n}(X) / z \cdot H^{n-p}(X)+\langle u, z\rangle \cdot H^{n-p-2 q+1}+\cdots \\
+\langle u, z\rangle_{k-1} \cdot H^{n-p-(k-1) q+1}(X), \\
n=q+k \cdot(p+q-1)
\end{aligned}
$$

as well-defined elements of these groups.

Proof. We make full use of the theory of principal fibre spaces, for which we refer to [7] [8]. The universal example, $(E, F, B ; p)$ may be considered a principal fibre space, with maps:

$$
\begin{aligned}
& \mu: E \times F \longrightarrow E \\
& m: F \times F \longrightarrow F .
\end{aligned}
$$

As in section 20 of [2], $E \times F$ is a fibre space over $B$, with projection map $\bar{p}: E \times F \longrightarrow B$ given by $\bar{p}(e, f)=p(e)$. Denote the spectral sequence of this fibre space by $\left\{\bar{E}_{r} ; \bar{d}_{r}\right\}$.

Then, we have

$$
\bar{E}_{r}=E_{r} \otimes H^{*}(F)^{4}
$$

$\bar{d}_{r}$ is $d_{r}$ on the first factor, and null on the second.

$$
\mu^{*}: E_{2} \longrightarrow \bar{E}_{2}
$$

is given by $1 \otimes m^{*}$.

$H^{*}(F)$ is either an exterior algebra on an odd-dimensional generator, or a polynomial algebra on an even-dimensional generator.

In the first case,

$$
m^{*}(x)=x \otimes 1+1 \otimes x,
$$

while in the second

$$
m^{*}\left(x^{k}\right)=\sum_{\substack{0 \leq i, j \leq k \\
i+j=k}}\left(\begin{array}{l}
k \\
i
\end{array}\right) x^{i} \otimes x^{j}
$$

where no repeats are taken in the sum. These facts follow immediately from the knowledge of $H^{*}(F)$, see [3], and duality. Hence, in $E_{2}$,

${ }^{4}$ We take the spectral sequence with real coefficients. 


$$
\mu^{*}\left(z \otimes x^{k}\right)=z \otimes m^{*}\left(x^{k}\right)=z \otimes\left(\sum_{\substack{0 \leq i, j \leq k \\
i+j=k}}\left(\begin{array}{l}
k \\
i
\end{array}\right) x^{i} \otimes x^{j}\right) \in \bar{E}_{2} .
$$

We will now show that there are non-zero real numbers $\alpha_{i},{ }_{j}$, so that if $\left\{z \otimes x^{k}\right\}$ represents the class in $H^{*}(E)$,

$$
\mu^{*}\left(\left\{z \otimes x^{k}\right\}\right)=\sum_{\substack{0 \leq i, j \leq k \\ i+j=k}} \alpha_{i, j}\left(\left\{z \otimes x^{i}\right\} \otimes x^{j}\right) \in H^{*}(E \times F) .
$$

First, notice that this relation with $\alpha_{i, j} \equiv 1$ is valid in $E_{\infty}$, because of the properties of the $\bar{d}_{r}$. Now $E_{\infty}$ and $\bar{E}_{\infty}$ are graded groups of $H^{*}(E)$ and $H^{*}(E \times F)$, with respect to certain filtrations. A homogeneous element of $\bar{E}_{\infty}$ has a degree with respect to the fibre, the dimension of the second part in $\bar{E}_{\infty}=E_{\infty} \otimes H^{*}(F)$. Furthermore, a homogeneous element in $\bar{E}_{\infty}$ and a representative for this element in $H^{*}(E \times F)$ have the same degree with respect to the fibre.

Corresponding to each degree in the fibre, in which an $x^{i}, i \leqq k$ lies, the term in $\bar{E}_{\infty}$ and the term in $H^{*}(E \times F)$ are both 1-dimensional vector spaces over $R$. In $\bar{E}_{\infty}$, each term in the sum which is

$$
\mu^{*}\left(\left\{z \otimes x^{k}\right\}\right)
$$

has a different degree with respect to the fibre. Hence, in $H^{*}(E \times F)$, the corresponding sum consists of distinct non-zero elements, which each have different degrees with respect to the fibre. As each $\left\{z \otimes x^{i}\right\} \otimes x^{j}$ is a non zero element in the corresponding 1-dimensional vector space, there exist non-zero $\alpha_{i, j}$ such that the desired relation holds. It is easy to see that $\alpha_{k, 1}=1$.

To prove the proposition, consider two maps $v, v^{\prime}: X \rightarrow E$ such that $p \cdot v=p \cdot v^{\prime}$. By Lemma 1.2 of [8], $\exists w: X \rightarrow F$ such that $\mu_{\sharp}(v, w)=v^{\prime}$. Then

$$
\begin{aligned}
v^{\prime}\left(\left\{z \otimes x^{k}\right\}\right) & =\mu_{\sharp}(v, w)^{*}\left(\left\{z \otimes x^{k}\right\}\right) \\
& =(v, w)^{*}\left(\mu^{*}\left(\left\{z \otimes x^{k}\right\}\right)\right. \\
& =(v, w)^{*}\left(\sum_{i+j=k} \alpha_{i, j}\left\{z \otimes x^{i}\right\} \otimes x^{j}\right) \\
& =v^{*}\left(\alpha_{k, 1}\left\{z \otimes x^{k}\right\} \cup 1\right)+\sum_{\substack{i+j=k \\
j<k}} \alpha_{i, j} v^{*}\left\{z \otimes x^{i}\right\} \cup w^{*}\left(x^{j}\right) \\
& =v^{*}\left(\left\{z \otimes x^{k}\right\}\right)+\sum_{\substack{i+j=k \\
j<k}} \alpha_{i, j} v^{*}\left(\left\{z \otimes x^{i}\right\}\right) \cup w^{*}\left(x^{j}\right) .
\end{aligned}
$$

Hence, by choosing different maps $v, v^{\prime},\langle u, z\rangle_{k}$ varies by at most an element of

$$
z \cdot H^{n-p}(X)+\cdots+\langle u, z)_{k-1} \cdot H^{n-p-(k-1) q+1}(X) .
$$

where $n=q+k \cdot(p+q-1)$. Therefore, $\langle u, z\rangle_{k}$ is a well-defined element of the stated group, completing the proof. 
REMARK. Using the same technique, a similar result may be established for the generalized triple product.

4. We now give some classification theorems for operations which go from integral cohomology to real cohomology.

THEOREM 4.1. Let $u_{1}, \cdots, u_{n}$ be classes which satisfy one, nontrivial, homogeneous relation $P\left[u_{1}, \cdots, u_{n}\right]=0$. Then, all the secondary operations which are defined under those circumstances are given by the generalized double products. Precisely, in each dimension, the term $E_{\infty}$ in the spectral sequence for the universal example is spanned by $p^{*}\left(H^{*}(B)\right)$ and the generalized double products.

Proof. The universal example here is a fibre space $(E, F, B ; p)$ with

$$
\begin{aligned}
& B=K\left(Z, \operatorname{dim} . u_{1}\right) \times \cdots \times K\left(Z, \operatorname{dim} . u_{n}\right) \\
& F=K(Z, \operatorname{dim} . P-1) .
\end{aligned}
$$

As before, take $u_{1}, \cdots, u_{n}$ as generators of $H^{*}(B)$ and $x$ as a generator of $H^{*}(F)$, with $\tau(x)=P\left[u_{1}, \cdots, u_{n}\right]$. We consider three cases.

Case 1. dim. $u_{i}$ is even for all $i$. We will show that there are no non-trivial secondary operations. As $\operatorname{dim} x$ is odd, there are only 2 nonzero rows in the $E_{2}$-term of the spectral sequence of $(E, F, B ; p)$. The elements are of the form $R \otimes 1$ or $R \otimes x$, where $R$ is a polynomial in the $u_{i}$. Clearly $d_{i}(R \otimes 1)=0$ for all $i$, and these terms respresent $P^{*}\left(H^{*}(B)\right)$. Now,

$$
\begin{gathered}
d_{i}(R \otimes x)=0, \text { if } \\
i<\operatorname{dim} . P=k, d_{k}(R \otimes x)=R \cdot P \otimes 1 .
\end{gathered}
$$

But $R \cdot P$ is not zero, unless $R=0$.

Case 2.

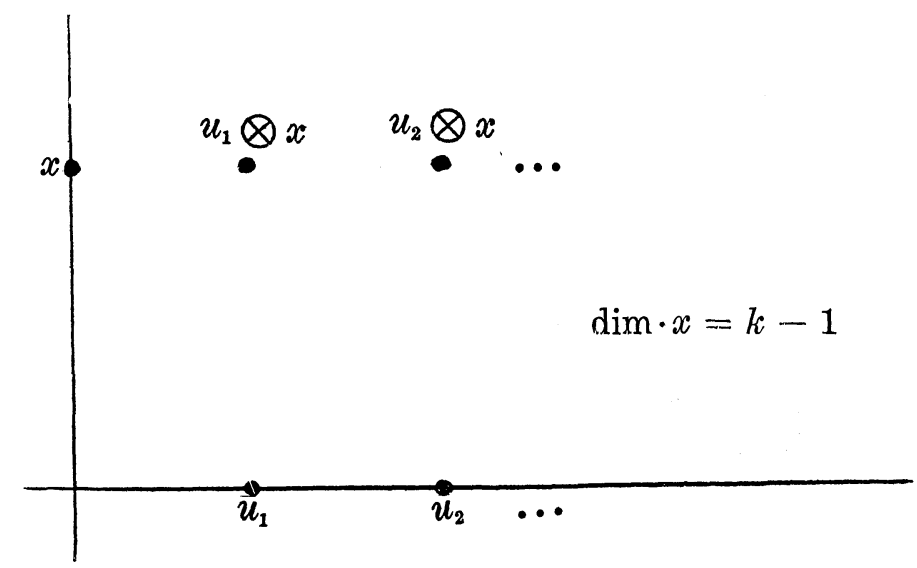


Suppose dim. $P$ is even, admitting the possibility of some $u_{i}$ have odd dimension. Again we have 2 non-zero rows in the $E_{2}$-term.

If $d_{k}(R \otimes x)=R \cdot P \otimes 1=0$ (recall $k=\operatorname{dim} . P$ ), then $\{R \otimes x\}$ remains until $E_{\infty}$, and represents the operation $\langle P \mid R, R\rangle$.

Case 3. dim. $P$ is odd, so that dim. $x$ is even. In this case, the $E_{2}$-term has infinitely many, non-zero rows.

Suppose that we have, in $E_{2}$ a homogeneous element of dim. $m$,

$$
u=R_{0}+R_{1} \otimes x+\cdots+R_{n} \otimes X^{n} ; \operatorname{dim} . R_{i}=m-i(k-1),
$$

which is a cocycle for each $d_{i}$, i.e., remains until $E_{\infty}$

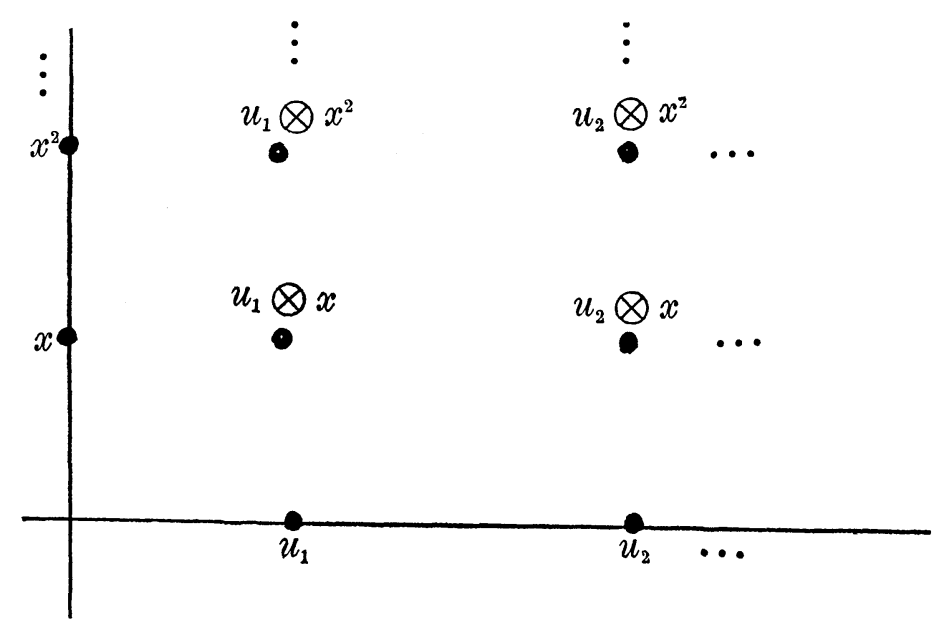

By assumption

$$
d_{i} u=0,2 \leqq i<k
$$

and

$$
d_{k} u=R_{1} \cdot P \otimes 1+2 R_{2} \cdot P \otimes x+\cdots+n \cdot R_{n} \cdot P \otimes x^{n-1}=0 .
$$

As each term has a different complementary degree, we have

$$
R_{1} \cdot P=0, \cdots, R^{n} \cdot P=0 .
$$

Now, $R_{0}$ and $R_{1} \otimes x$ represent cocycles for each $d_{i}$; we have

$$
d_{i}\left(R_{2} \otimes x^{2}+\cdots+R_{n} \otimes x^{n}\right)=0 \text {, all } i,
$$

and in the same way.

$$
d_{i}\left(R_{2} \otimes x^{2}\right)=0, \text { all } i
$$


Hence, proceeding in this way, we see that each term, $R_{i} \otimes x^{i}$, remains until $E_{\infty}$. Therefore, $R_{i} \otimes x^{i}$ represents a double product $\left\langle p / R_{i}, R_{i}\right\rangle_{i}$, or

$$
\{u\}=R_{0}+\left\langle p / R_{1}\right\rangle_{1}+\cdots+\left\langle p / R_{n}, R_{n}\right\rangle_{n} .
$$

We state the following similar theorem without proof ; the proof is complicated.

Theorem 4.2. Consider operations in 3 variables $u, v, w$, defined on the kernel $\{u, v, w / u \cdot v=0, v \cdot w=0\}$. All such operations are given (in the sense of the above theorem) by sums of products of (possibly generalized) double and triple products.

REMARK. In either of the above theorems, if there is exactly one element in a given dimension in $E_{\infty}$, then the operation involved, as a class in $H^{*}(E)$ is determined exactly.

The following theorem of Massey may be proven either by these methods, or by using the Gysin sequence.

Theorem 4.3. (Massey). Consider $n$ even-dimensional cohomology classes which satisfy two distinct, non-trivial, polynomial relations.

$$
\begin{aligned}
& f\left(u_{1}, \cdots, u_{n}\right)=0 \\
& g\left(u_{1}, \cdots, u_{n}\right)=0 .
\end{aligned}
$$

Let $h$ be the greatest common divisor of $f$ and $g$, over the reals.

$$
f=h \cdot f_{1} \quad g=h \cdot g_{1} .
$$

Then, $\left\langle f_{1}, h, g_{1}\right\rangle$ generates all secondary operations which are then defined, as a module over the primary operations.

\section{BIBLIOGRAPHY}

1. J. F. Adams, On the Non-Existense of Elements of Hope Invariant One, Annals of Math., (72) 1960.

2. A. Borel, Sur la Cohomologie des Espaces Fibrés Principeaux...Annals of Math., (57) 1953.

3. S. Eilenberg and S. MacLane, On the Groups $H(\pi, n) I I I$, Annals of Math., (60) 1954.

4. D. W. Kahn, On the existence and applications of anti-commutative cochain algebras, Illinois J. Math., (to appear).

5. W. S. Massey and H. Uehara, The Jacobi Identity for Whitehead Products, Alg. Geometry and Topology, Princeton University Press.

6. W. S. Massey, On the Cohomology Ring of a Sphere Bundle, Jour. of Math. and Mech.

(7) 1958.

7. F. P. Peterson and N. Stein, Secondary operations ; Two formulas, Amer. J. Math., (81) 1959.

8. F. P. Peterson and E. Thomas, A Note on Non-Stable Cokomology Operations, Bol. Soc. Mat. Mexican 1958.

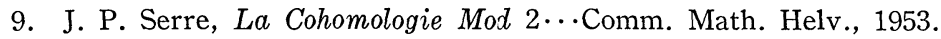

10. - Homologie Singulière des Espaces Fibrés, Annals of Math. (54) 1951. 



\section{PACIFIC JOURNAL OF MATHEMATICS}

\section{EDITORS}

\section{RalPh S. Phillips}

Stanford University

Stanford, California

M. G. Arsove

University of Washington

Seattle 5, Washington
J. Dugundji

University of Southern California Los Angeles 7, California

Lowell J. Paige

University of California

Los Angeles 24, California

\section{ASSOCIATE EDITORS}
E. F. BECKENBACH
D. DERRY
H. L. ROYDEN
E. G. STRAUS
T. M. CHERRY
M. OHTSUKA
E. SPANIER
F. WOLF

\section{SUPPORTING INSTITUTIONS}

\author{
UNIVERSITY OF BRITISH COLUMBIA \\ CALIFORNIA INSTITUTE OF TECHNOLOGY \\ UNIVERSITY OF CALIFORNIA \\ MONTANA STATE UNIVERSITY \\ UNIVERSITY OF NEVADA \\ NEW MEXICO STATE UNIVERSITY \\ OREGON STATE UNIVERSITY \\ UNIVERSITY OF OREGON \\ OSAKA UNIVERSITY \\ UNIVERSITY OF SOUTHERN CALIFORNIA
}

STANFORD UNIVERSITY

UNIVERSITY OF TOKYO

UNIVERSITY OF UTAH

WASHINGTON STATE UNIVERSITY

UNIVERSITY OF WASHINGTON

AMERICAN MATHEMATICAL SOCIETY

CALIFORNIA RESEARCH CORPORATION SPACE TECHNOLOGY LABORATORIES

NAVAL ORDNANCE TEST STATION

Printed in Japan by International Academic Printing Co., Ltd., Tokyo Japan 


\section{Pacific Journal of Mathematics}

\section{Vol. 13, No. 1 \\ March, 1963}

Frantz Woodrow Ashley, Jr., A cone of super-(L) functions............. 1

Earl Robert Berkson, Some metrics on the subspaces of a Banach space....

Felix Earl Browder and Walter Strauss, Scattering for non-linear wave

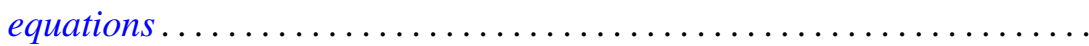

Edmond Darrell Cashwell and C. J. Everett, Formal power series ..........

Frank Sydney Cater, Continuous linear functionals on certain topological

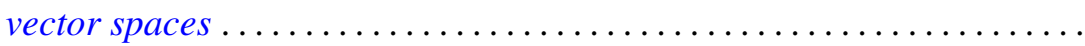

John Douglas Dixon, General group extensions ....................

Robert Pertsch Gilbert, On harmonic functions of four variables with

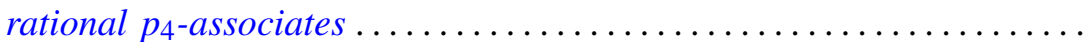

Irving Leonard Glicksberg, On convex hulls of translates ..............

Simon Hellerstein, On a class of meromorphic functions with deficient zeros

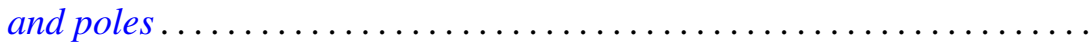

Donald William Kahn, Secondary cohomology operations which extend the

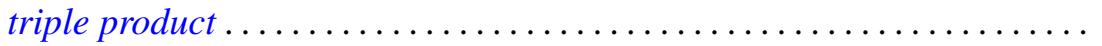

G. K. Leaf, A spectral theory for a class of linear operators .............

R. Sherman Lehman, Algebraic properties of the composition of solutions of partial differential equations ........................... 157

Joseph Lehner, On the generation of discontinuous groups ............. 169

S. P. Lloyd, On certain projections in spaces of continuous functions ...... 171 Fumi-Yuki Maeda, Generalized spectral operators on locally convex spaces ..................................

Donald Vern Meyer, $E^{3}$ modulo a 3-cell

William H. Mills, An application of linear programming to permutation groups.

Richard Scott Pierce, Centers of purity in abelian groups

Christian Pommerenke, On meromorphic starlike functions ...

Zalman Rubinstein, Analytic methods in the study of zeros of

polynomials...

B. N. Sahney, On the Nörlund summability of Fourier series

Tôru Saitô, Regular elements in an ordered semigroup . .

Lee Meyers Sonneborn, Level sets on spheres...........

Charles Andrew Swanson, Asymptotic estimates for limit point

problems .

Lucien Waelbroeck, On the analytic spectrum of Arens . .

Alvin (Murray) White, Singularities of a harmonic function of three

variables given by its series development .............

Kōichi Yamamoto, Decomposition fields of difference sets ...

Chung-Tao Yang, On the action of $\mathrm{SO}(3)$ on a cohomology manifold... 